\title{
Estrutura demográfica de regeneração natural de palmeiras em fragmentos florestais na Amazônia Oriental
}

O objetivo desse trabalho foi comparar a estrutura da regeneração natural de palmeiras de fragmentos florestais nas comunidades de Agrovila Araipá e São Tomé no município de Aveiro (PA). Foi realizado o método de transecção em linha, sendo quatro transecções: duas em cada comunidade e distribuídos em dois ecossistemas diferentes: um em ecossistemas de floresta primária remanescente e outro em ecossistema de floresta secundária. Todos os indivíduos não reprodutivos da parcela foram registrados, sendo classificados em seis estádios de vida, mas apenas os quatros primeiros estádios foram inclusos no estudo. Cada transecção media $300 \mathrm{~m}$ de comprimento e $10 \mathrm{~m}$ de largura dentro de cada fragmento. Na floresta primária, foram 390 indivíduos distribuídos em duas espécies (Astrocaryum aculeatum Meyer e Attalea maripa (Aublet) Martius) e 226 indivíduos distribuídos em três (Attalea sp., Astrocaryum aculeatum Meyer e Attalea maripa (Aublet) Martius) espécies na floresta secundária. A análise da estrutura revelou alta ocorrência de indivíduos da espécie $A$. maripa em estádios iniciais de desenvolvimento, causa essa provável pela ausência e/ou ineficiência dos dispersores dessa espécie. A espécie $A$. aculeatum, não apresentou diferença em sua ocorrência nos dois tipos de florestas (primária e secundária). Entretanto, na floresta secundária, a ocorrência de Attalea sp. foi considerada relevante na área ocorrendo exclusivamente nessa vegetação; isso, possivelmente porque a floresta primária está fragmentada, cercada de pastagens e plantios agrícolas, causando um desiquilíbrio no recrutamento da $A$. maripa, sendo a provável uma característica da espécie uma alta produção de frutos e consequentemente um maior número de indivíduos em estádios iniciais de vida.

\section{Demographic Structure of Natural Regeneration of Palm Trees in Forest Fragments in the Eastern Amazon}

\begin{abstract}
The objective of this work was to compare the structure of palm tree natural regeneration in the communities of Agrovila Araipá and São Tomé in Aveiro (PA). The inline transection method was performed, being four transections: two in each community and distributed in two different ecosystems: one in remnant primary forest ecosystems and another in secondary forest ecosystem. All non-reproductive individuals in the plot were registered and classified into six stages of life, but only the first four stages were included in the study. Each transection measured 300m long and $10 \mathrm{~m}$ wide within each fragment. In the primary forest, 390 individuals were distributed in two species (Astrocaryum aculeatum Meyer and Attalea maripa (Aublet) Martius) and 226 individuals distributed in three (Attalea sp., Astrocaryum aculeatum Meyer and Attalea maripa (Aublet) Martius) species in the secondary forest. The structure analysis revealed a high occurrence of A. maripa individuals in the early stages of development, probably due to the absence and / or inefficiency of the dispersers of this species. The species A. aculeatum showed no difference in its occurrence in both types of forests (primary and secondary). However, in the secondary forest, Attalea sp. was considered relevant in the area, occurring exclusively in this vegetation; This is possibly because the primary forest is fragmented, surrounded by pasture and cropland, causing an imbalance in A. maripa recruitment. A high yield of fruit is likely to be a characteristic of the species and, consequently, a larger number of individuals in the early stages life.
\end{abstract}

Keywords: Remaining primary forest; Secondary forest; Attalea genus; Astrocaryum aculeatum; Stages of development.

Topic: Ciências Florestais

Reviewed anonymously in the process of blind peer.
Received: 03/08/2018

Approved: 16/08/2018
Danielly Caroline Miléo Gonçalves (iD)

Universidade Federal do Oeste do Pará, Brasil

http://lattes.cnpq.br/5083066032365508

http://orcid.org/0000-0003-0521-115X

daniellycmg@gmail.com

João Ricardo Vasconcellos Gama (DD

Universidade Federal do Oeste do Pará, Brasil

http://lattes.cnpq.br/9058536716453750

http://orcid.org/0000-0002-3629-3437

irvgama@gmail.com

Raimundo Cosme de Oliveira Júnior

Universidade Federal do Oeste do Pará, Brasil

http://lattes.cnpq.br/1210121065910515

raimundo.oliveira-junior@embrapa.br

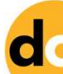

DOI: 10.6008/CBPC2179-6858.2018.006.0005
Referencing this:

GONÇALVES, D. C. M.; GAMA, J. R. V.; OLIVEIRA JÚNIOR, R. C.. Estrutura demográfica de regeneração natural de palmeiras em fragmentos florestais na Amazônia Oriental. Revista Ibero-Americana de Ciências Ambientais, v.9, n.6, p.44-51, 2018. DOI: http://doi.org/10.6008/CBPC2179-6858.2018.006.0005 


\section{INTRODUÇÃO}

O estudo da regeneração natural permite prever o comportamento e desenvolvimento futuro da floresta, fornecendo informação sobre a relação e a quantidade de espécies que constituem seu estoque, bem como suas dimensões e distribuição na área (MARANGON et al., 2008). Portanto, a regeneração natural decorre da interação de processos naturais de restabelecimento do ecossistema florestal, assim sendo, parte do ciclo de crescimento da floresta e se refere às fases iniciais de seu estabelecimento e desenvolvimento (GAMA et al., 2002; MARANGON et al., 2008).

Na Amazônia, a importância das palmeiras é documentada em diversos estudos etnobotânicos, em relação aos aspectos alimentar, medicinal ou socioeconômico (BALICK, 1984; KAHN et al., 1992; HENDERSON et al., 1995; JARDIM et al., 1994; JARDIM et al., 1998; MIRANDA et al., 2001; VALENTE, 2001). Seu manejo tem se tornado de interesse crescente, pois são espécies resistentes, principalmente ao desmatamento e queimadas (MIRANDA et al., 2001). No entanto, os estudos de regeneração natural de populações de palmeiras são insuficientes na Amazônia, portanto pouco se conhece da estrutura e dinâmica populacional de dessas espécies (PORTELA et al., 2011).

O gênero Attalea registra 33 espécies catalogadas, dentre elas Attalea maripa (Aublet) Martius e Attalea speciosa Martius, cujas distribuições são amplas, alcançando quase todos os estados da região Norte, como também os das regiões Centro Oeste e Nordeste (LEITMAN et al., 2013). Possuindo domínios fitogeográficos na Amazônia ocorrendo em áreas antrópicas, campinarana, floresta de terra firme e de várzea, com alta plasticidade, tendo uma ampla ocorrência em diferentes ambientes, desde matas fechadas a pastos (ARAÚJO et al., 2012). Seu fruto é fonte alimentar para fauna das florestas e tem um alto potencial oleaginoso para uso como biodiesel (MATOS, 2010).

A A. maripa ocorre em áreas de florestas primárias e secundárias, campos naturais e cerrados, principalmente nas áreas alteradas pelo homem, em especial as de pastagens, e que passaram por um processo de queimada. Embora tolere áreas alagadiças, ela é mais adaptada aos lugares com solos bem drenados (FAO, 1998). É tolerante a queimadas, pois, as plantas jovens, ao serem queimadas para o cultivo de pastagens, rebrotam com vigor e as sementes, que estavam em processo de dormência, germinam rapidamente (LORENZI et al., 2010).

A palmeira Astrocaryum aculeatum Meyer, conhecida popularmente como tucumã, é comum na floresta densa da Amazônia central onde domina ocasionalmente florestas secundárias (SALM, 2004). Sua importância econômica baseia-se principalmente na exploração da polpa dos frutos, que pode ser consumida ao natural ou na forma de sorvete, suco, licor e doce (DIDONET et al., 2014). Da polpa dos frutos e das sementes, podem ser extraídos diferentes tipos de óleos comestíveis, além de poderem ser utilizadas na suplementação alimentar humana e na fabricação de ração animal (GENTIL et al., 2005). Tem ocorrência no Acre, Amazonas, Rondônia, Pará, Mato Grosso e Roraima, desde sistemas agroflorestais às plantas invasoras de pastagens, permitindo assim seu aproveitamento (LORENZI et al., 2004).

Considerando que no processo inicial de sucessão florestal, após desflorestamento, as palmeiras se colonizam e se estabelecem na fitocenose de forma mais abundante, como também sua importância ecologia 
e socioeconômica para povos e comunidades tradicionais (MIRANDA et al., 2001), o objetivo deste trabalho foi comparar a estrutura da regeneração natural das comunidades de palmeiras, Attalea sp., Attalea maripa (Aubl.) Mart. e Astrocaryum aculeatum Mey. em floresta primária remanescente e floresta secundárias.

\section{MATERIAIS E MÉTODOS}

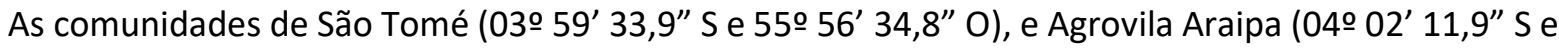
55 55' 06" W) estão localizadas na margem direita do rio Tapajós, em Aveiro, Pará (figura 1). Os ecossistemas dominantes são florestas em diferentes estágios serais (capoeiras), agroecossistemas de pastagem, com predominância de Paspalum spp. e Brachiaria humidicola (quicuio), e de subsistência como a Manihot sativa L. (mandioca) e Zea mayz L. (milho) (GONÇALVES, 2008). Existem, nessa região, áreas de ecossistemas de florestas primárias remanescentes onde os comunitários utilizam recursos da floresta para consumo próprio e de sua família.

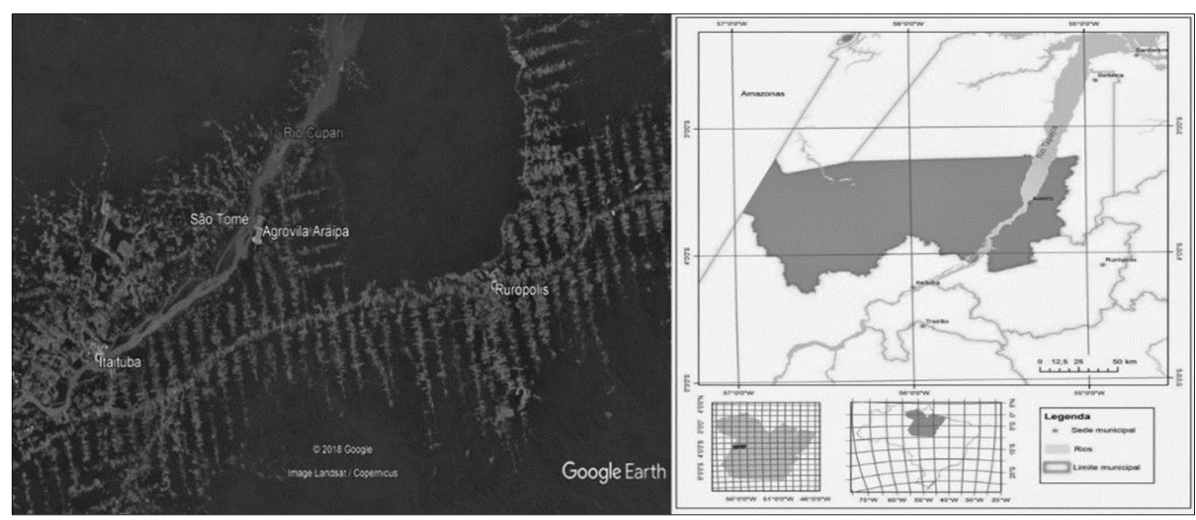

Figura 1: Comunidades de Agrovila Araipa e São Tomé, Aveiro (PA).

São Tomé é uma comunidade tradicional composta por pescadores e agricultores que utilizam vários produtos das palmeiras (folhas para cobertura de casas, fruto, entre outros), enquanto que Araipa, em sua maioria é composta por agricultores, destacando-se os cultivos de milho (Zea mays), arroz (Oryza sp.) e mandioca (Manihot esculenta) (BÉLIVEAU, 2007). A colonização de Araipa ocorreu após a criação da rodovia Transamazônica, por agricultores oriundos de vários Estados do Nordeste brasileiro (Ceará, Paraíba, Maranhão, etc.) e de outras regiões do país, representando $47 \%$ do total da população (VALADÃO, 2009).

O relevo é do tipo tabular, com rebordas erosivas e trechos com declividades fortes ou moderadas (MME, 1976; ESPÍRITO-SANTO et al., 2005). O solo é do tipo Latossolo Amarelo Distrófico, caracterizado por diferentes texturas, geralmente profundo, ácido, friável e revestido por florestas densas. Os Argissolos Vermelho-Amarelo também ocupam grande porção da área, nas partes baixas dos vales é comum a presença de Neossolos Flúvicos típicos e, em menor proporção, Gleissolos Háplicos (MME, 1976; WRB, 2006).

A temperatura média anual é de $25,6^{\circ} \mathrm{C}$ e valores médios para a máxima de $31^{\circ} \mathrm{Ce}$, para a mínima de $22,5^{\circ} \mathrm{C}$. O tipo climático é Ami, segundo a classificação Köppen. Quanto à umidade relativa, apresenta valores acima de $80 \%$, em quase todos os meses do ano. A pluviosidade aproxima-se dos $2.000 \mathrm{~mm}$ anuais. As estações chuvosas são entre os meses de dezembro a junho (mais chuvosa) e as menos chuvosas de julho a novembro (SABLAYROLLES et al., 2009). 
Foi realizado o método de transecção em linha (BROWER et al., 1984). Foram instaladas quatro transecções, sendo dois em cada comunidade (dois em São Tomé e dois em Araipa), e distribuídos em dois ecossistemas diferentes, um em ecossistemas de florestas primárias remanescentes e outro em ecossistemas sucessionais (capoeira), variando de 3 a 6 anos de idade. Cada transecção media 300m de comprimento e $10 \mathrm{~m}$ de largura, dentro de cada fragmento. As áreas amostradas têm visualmente inclinação do terreno e umidade de solos similares para que sejam evitados efeitos diversos nas populações, evitando áreas brejosas ou muito inclinados (SCARIOT, 1999; PORTELA et al., 2011).

Para o estudo da estrutura demográfica da regeneração, todos os indivíduos não reprodutivos da parcela foram registrados, sendo classificados em seis estádios de vida, conforme dados na tabela 1. Assim, foram registrados para esse estudo, apenas os quatros primeiros estádios, correspondendo aos estágios plântula, jovem pré-estabelecido, jovem novo estabelecido, e jovem médio estabelecido. Todos os indivíduos da pesquisa não apresentavam estipe aparente (sem estipe acima do solo ou este coberto por bainhas foliares).

Portanto, a altura foi medida da altura da coroa foliar até a parte aparente sobre o solo, foram também observadas às folhas de cada indivíduo, sendo diferenciada a partição do limbo foliar, podendo ser inteiro, pinatissecto ou transicional (parte inteira e parte pinatissecta), a presença ou não de folhas mortas e a presença ou não de matéria orgânica na coroa foliar. Essas informações serviram para classificar os indivíduos em estádios de desenvolvimento (GATSUK et al., 1980). Foi utilizado ANOVA one-way, para comparar se há diferença na densidade das espécies nos dois diferentes ecossistemas. Os dados foram processados no Office Excel 2016.

Tabela 1: Definições dos seis (6) estádios de desenvolvimento de palmeiras.

\begin{tabular}{|l|l|l|}
\hline $\begin{array}{l}\mathbf{N}^{\circ} \text { de } \\
\text { Estádios }\end{array}$ & Estádio & Definição \\
\hline Estádio 1 & Plântula & Primeiras folhas oriundas da germinação ainda não divididas \\
\hline Estádio 2 & $\begin{array}{l}\text { Jovem pré- } \\
\text { estabelecido }\end{array}$ & Primeiras divisões do limbo foliar, a folha não é totalmente dividida \\
\hline Estádio 3 & $\begin{array}{l}\text { Jovem novo } \\
\text { estabelecido }\end{array}$ & $\begin{array}{l}\text { Numerosas folhas completamente divididas sem estipe aéreo e com gema terminal } \\
\text { ainda dentro do chão }\end{array}$ \\
\hline Estádio 4 & $\begin{array}{l}\text { Jovem médio } \\
\text { estabelecido }\end{array}$ & $\begin{array}{l}\text { Numerosas folhas completamente divididas sem estipe aéreo e com gema terminal } \\
\text { nas superfícies }\end{array}$ \\
\hline Estádio 5 & Adulto estéril & $\begin{array}{l}\text { Estipe livre (geralmente de menos de 3 metros) sem presença ou traço de órgãos } \\
\text { reprodutores. }\end{array}$ \\
\hline Estádio 6 & Adulto fértil & $\begin{array}{l}\text { Estipe livre (geralmente maior de 3 metros) com presença e/ou traço de órgãos } \\
\text { reprodutores. }\end{array}$ \\
\hline
\end{tabular}

Fonte: Gatsuk et al. (1980).

\section{RESULTADOS}

Ao todo, foram registradas 794 palmeiras, 568 indivíduos foram encontrados em floresta primária remanescente, sendo apenas das espécies Atallea maripa e Astrocaryum aculeatum e em floresta secundária foram 226 indivíduos, distribuídos em três espécies: Attalea sp. Atallea maripa e Astrocaryum aculeatum. A A. maripa (Inajá) apresentou maior densidade com cerca de 87 indivíduos/ha nos dois tipos de vegetação e frequência de $100 \%$ em todas as 12 parcelas. 
A classificação em estádios de desenvolvimento (figura 2) mostra que, o A. maripa possui maior número de indivíduos nos estágios iniciais de desenvolvimento, sendo, mas expressivo nas florestas primárias (307 indivíduos). Como essas áreas são fragmentadas, acredita-se que a existência de clareiras, beneficie a germinação de suas sementes. Outro ponto observado em campo é a existência de muitas sementes próxima à planta-mãe, indicando a ausência ou ineficiência dos dispersores (LORENZI et al., 2010).

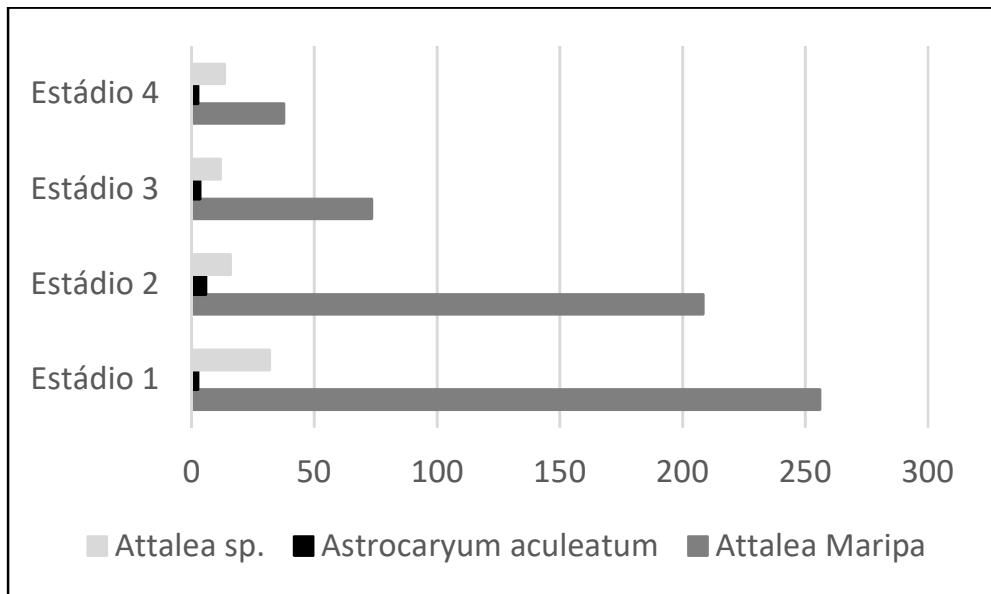

Figura 2: Número de palmeiras por estádios de desenvolvimento nas comunidades de São Tomé e Agrovila Araipa, Aveiro, (PA).

A distribuição das espécies em relação ao tipo de vegetação demonstrou que não possui diferença significativa, segundo a ANOVA $\left(F=0,008 ; p<0,93, F_{\text {crit }}=7,70\right)$ confirmado também pelo teste $t$ de duas amostras presumindo variância equivalente (tabela 2). Portanto, a densidade das espécies não tem diferenças entre as áreas, apesar de serem vegetação diferentes, a proximidade da comunidade humana da floresta primária, principalmente pela existência pastagens, plantios agrícolas e também da exploração eventual de espécies vegetais para outros fins, estabelece uma característica comum a floresta secundária, a abertura de clareiras, possibilitando que espécies primárias se estabelecem no local dos espaços aberto da floresta.

Tabela 2: Teste $t$ de duas amostras de comparação de médias ordenadas, para a densidade das três espécies de palmeiras em relação à diferença de vegetação.

\begin{tabular}{|l|c|c|l|}
\hline \multicolumn{1}{|c|}{$\#$} & FP & FS & \\
\hline Média & 7,96 & 7,29 & Média \\
\hline Variância & 141,64 & 14,43 & Variância \\
\hline Observações & 3,00 & 3,00 & Observações \\
\hline Variância agrupada & 78,03 & & Variância agrupada \\
\hline Hipótese da diferença de média & 0,00 & & Hipótese da diferença de média \\
\hline GI & 4,00 & & Gl \\
\hline Stat t & 0,09 & & Stat $\mathrm{t}$ \\
\hline $\mathrm{P}(\mathrm{T}<=\mathrm{t})$ uni-caudal & 0,47 & & $\mathrm{P}(\mathrm{T}<=\mathrm{t})$ uni-caudal \\
\hline $\mathrm{t}$ crítico uni-caudal & 2,13 & & $\mathrm{t}$ crítico uni-caudal \\
\hline $\mathrm{P}(\mathrm{T}<=\mathrm{t})$ bi-caudal & 0,93 & & $\mathrm{P}(\mathrm{T}<=\mathrm{t})$ bi-caudal \\
\hline $\mathrm{t}$ crítico bi-caudal & 2,78 & & $\mathrm{t}$ crítico bi-caudal \\
\hline
\end{tabular}

Legenda: FP: Floresta primária; FS: Floresta secundária.

\section{DISCUSSÃO}

Nos dois tipos de vegetação do estudo, a maior frequência foi da espécie $A$. maripa, ocorrendo principalmente nos primeiros estádios de desenvolvimento. Matos (2010) constatou em seu trabalho que $A$. 
maripa é comum em pastagem e roçado em pousio, formando agrupamentos conhecidos por inajazais, característica também apresentada no presente estudo. A alta densidade encontrada de A. maripa em floresta primária, está relacionada com a ação antrópica que favorece sua distribuição, devido à abertura de dossel, aumenta a incidência de luz no sub-bosque, promovendo seu estabelecimento (SALM, 2004). Outra razão para alta incidência de plântulas é que $A$. maripa não possui dispersores eficientes para a propagação de suas sementes, e essa, por sua vez, acaba germinando próximo da planta mãe (ARAÚJO et al., 2012).

A Attalea sp. não teve ocorrência na floresta primária; apenas na floresta secundária com 87 indivíduos, demonstrando adaptação dessa espécie em ambientes alterados, necessitando de ambientes abertos e com alta incidência de luz, quase que exclusivamente ocorre em solos argilosos de ótima fertilidade e ricos em cálcio (NEGRELLE, 2015). A espécie A. aculeatum, apresentou apenas 17 indivíduos nas áreas estudadas, sendo uma espécie de Floresta Ombrófila Aberta que se adapta bem as áreas aberta, assim como Attalea sp. e A. maripa (MIRANDA et al., 2001).

A utilização da classificação de Gatsuk et al. (1980) e Portela et al. (2011), para a definição dos estádios iniciais de desenvolvimento, onde o estádio é definido por atributos quantitativos e qualitativos, e geralmente são caracterizadas pelo aparecimento de estruturas que estavam ausentes em estádios mais recentes de desenvolvimento e pela perda de outras que estavam presentes.

$\mathrm{Na}$ análise da estrutura populacional, foi observado que a população apresentou o maior número de indivíduos concentrado nos estágios iniciais de desenvolvimento (Plântula e Jovem pré-estabelecido), sendo o número de indivíduos da primeira classe, expressivamente maior que o número de indivíduos dos demais estádios, principalmente de A. maripa. Trabalhos como de Bernacci et al. (2008), apontaram resultados parecidos com a população de jerivá (Syagrus romanzoffiana (Cham.) Glassman), que apresentou maior ocorrência de estádios iniciais; entretanto, foram, ao menos em parte, decorrentes de não terem sido observadas mortalidade nem transições para os estádios.

Estes dados podem indicam que a população está em crescimento (ANTONINI et al., 2004) e que os indivíduos dos estádios iniciais são aqueles que garantem a manutenção da população, embora estes sofram maiores taxas de mortalidade, enquanto os indivíduos mais desenvolvidos terão maior chance de se estabelecer e reproduzir no ambiente. Gama et al. (2002) cita que essa é a tendência esperada para as espécies que estão em equilíbrio dinâmico.

Não foi possível conhecer, no campo, a longevidade do estádio de plântulas de $A$. maripa, mas observou-se que a maioria das plântulas, não muda de estádio por mais de um ano e que o tempo de permanência, de acordo com a taxa de transição observada, é de cerca de três anos (BERNACCl et al., 2008). Pode-se considerar como sendo uma característica do gênero Attalea uma alta produção de fruto e consequentemente um alto índice de plântulas, já que a categoria mais numerosa e com longo tempo de persistência na população foi a dos indivíduos de folha inteira, na qual as plântulas estão incluídas, como ocorre no Jerivá (BERNACCl et al., 2008). 


\section{CONCLUSÕES}

Nas florestas primária e na sucessional tem uma maior ocorrência de palmeiras de $A$. maripa em estádios iniciais de vida. A espécie $A$. aculeatum, não apresentou diferença por fitocenose, ocorrendo igualmente nos dois tipos de ecossistemas. Na floresta secundária a ocorrência de Attalea sp., foi considerada relevante na área, ocorrendo exclusivamente nessa vegetação, portanto, é uma espécie especifica de vegetação sucessional. Na comparação de densidade das espécies e sua distribuição entre as duas florestas, não se obteve diferença significativa. Possivelmente, porque a floresta primária está fragmentada, cercada de pastagens e plantios agrícolas, causando um desiquilíbrio no recrutamento da $A$. maripa, sendo a provável característica da espécie, uma alta produção de frutos e consequentemente um maior número de indivíduos em estádios iniciais de vida.

\section{REFERÊNCIAS}

ANTONINI, R. D.; NUNES-FREITAS, A. F.. Estrutura populacional e distribuição espacial de Miconia prasina D. C. (Melastomataceae) em duas áreas de Floresta Atlântica na Ilha Grande, RJ, Sudeste do Brasil. Acta Botânica Brasílica, São Paulo, v.8, p.671-676, 2004. DOI: http://doi.org/10.1590/S0102-33062004000300024

ARAÚJO, G. C.; JUNIOR, R. C. O.; OLIVEIRA, F. A.; GAMA, J. R. V.; GONÇALVES, D. C. M.; ALMEIDA, L. S.. Comparação entre Floresta Primária e Secundária com Ocorrência de Attalea maripa (Aubl.) Mart.: estudo de caso na Amazônia Oriental. Floresta e Ambiente, v.19, n.3, p.325-335, 2012. DOI: http://doi.org/10.4322/floram.2012.038

BALICK, M. J.. Systematics and Economic Botany of the Oenocarpus-Jessenia (palmae) complex. Advances in Economic Botanic, New York, n.3, p.1-140, 1986.

BÉLIVEAU, A.. Déforestation et agriculture sur brûlis en Amazonie brésilienne: les impacts de la première année de culture sur les sols de fermes familiales de la région du Tapajós. Dissertação (Mestrado en Sciences de l'environnement) - Université Du Québec À Montréal, Montreal, 2007.

BERNACCI, L. C.; MARTINS, F. R.; SANTOS, F. A. M.. Dinâmica populacional da palmeira nativa jerivá, Syagrus romanzoffiana (Cham.) Glassman em um fragmento florestal no sudeste do Brasil. Acta Botânica Brasílica, Feira de Santana, v.22, n.1, p.119-130, 2008.

BROWER, J. E.; ZAR, J. H.. Field and laboratory methods for general ecology. 2 ed. Dubuque: William. C. Brown Publishers, 1984.

DIDONET, A. A.; FERRAZ, I. D. K.. O comércio de frutos de tucumã (Astrocaryum aculeatum G. Mey - arecaceae) nas feiras de Manaus (Amazonas, Brasil). Revista Brasileira Fruticultura, Jaboticabal, v.36, n.2, p.353-362, 2014. DOI: http://doi.org/10.1590/0100-2945-108/13

ESPIRITO-SANTO, F. D. B.; SHIMABUKURO, Y. E.; ARAGÃO, L. E. O. C.; MACHADO, E. L. M.. Análise da composição florística e fitossociológica da Floresta Nacional do Tapajós com apoio geográfico de imagem de satélite. Acta amazônica, v.2, n.35, p.155-173, 2005. DOI: http://doi.org/10.1590/S0044$\underline{59672005000200006}$

FAO. Organização das Nações Unidas para a Alimentação e a Agricultura. Tropical palms: Roma: Non-Wood Forest Products, 1998.

GAMA, J. R. V., BOTELHO, A. S.; BENTES-GAMA, M. M. R.. Composição florística e estrutura da regeneração natural de floresta secundária de várzea baixa no estuário amazônico. Revista Árvore, v.26, n.1, p.559-566, 2002.

GATSUK, L. E.; SMIRNOVA, O. V.; VORONTZOVA, L. I.; ZAUGOLNOVA, L. B.; ZHUKOVA, L. A.. Age states of plants of various growth forms: a review. Journal of Ecology, v.68, p.675-96, 1980.

GENTIL, D. F. O.; FERREIRA, S. A. N.. Morfologia da plântula em desenvolvimento de Astrocaryum aculeatum Meyer (Arecaceae). Acta Amazônica, v.35, n.3, p.337-342, 2005. DOI: http://doi.org/10.1590/S004459672005000300005

GONÇALVES, D. C. M.. Estrutura de ecossistemas florestais sucessionais e ecossistemas remanescentes com predomínio de Attalea maripa (Aubl.) Mart. e Astrocaryum aculeatum G. Mey e a comercialização de produtos nãomadeireiros na região do Baixo Rio Tapajós, Amazônia Oriental. Dissertação (Mestrado em Ciências Florestais) Universidade Federal Rural da Amazônia, Belém, 2008.

HENDERSON, A.; GALEANO, G.; BERNAL, R.. Field Guide to the Palms of the Americas. New Jersey: Princeton University Press, 1995.

JARDIM, M. A. G.; CUNHA, A. C. C.. Usos de palmeiras em uma comunidade ribeirinha do estuário amazônico. Boletim do Museu Paraense Emílio Goeldi, série botânica, v.14, p.69-77, 1998.

JARDIM, M. A. G.; STEWART, P. J.. Aspectos etnobotânicos e ecológicos de palmeiras no município de Novo Airão, Estado do Amazonas, Brasil. Boletim do Museu Paraense Emílio Goeldi, v.10, p.69-76, 1994. 
KAHN, F.; GRANVILLE, J. J.. Palms in forest ecosystems of Amazonia. Berlim: Springer Verlag, 1992.

LEITMAN, P.; HENDERSON, A.; NOBLICK, L.; MARTINS, R. C.. Arecaceae. Rio de Janeiro: JBRJ, 2013.

LORENZI, H.; NOBLICK, L.; KAHN, F.; FERREIRA, E.. Flora Brasileira Lorenzi: Arecaceae (palmeiras). Nova Odessa: IP, 2010.

LORENZI, H.; SOUZA, H. M.; COSTA, J. T. M.; CERQUEIRA, L. S. C.; FERREIRA, E.. Palmeiras brasileiras e exóticas cultivadas. Nova Odessa: IP, 2004.

MARANGON, L. C.; SOARES, J. J.; FELICIANO, A. L. P., BRANDÃO, C. F. S.. Regeneração natural em um fragmento de floresta estacional semidecidual em Viçosa, Minas Gerais. Revista Árvore, v.32, n.1, p.183-191, 2008. DOI: http://doi.org/10.1590/S0100-67622008000100020

MATOS A. K. M. G.. Biometria e morfologia de Attalea maripa (Aubl.) Mart. (inajá) em sistema silvopastoril no nordeste paraense. Dissertação (Mestrado em Ciências Florestais) - Universidade Federal Rural da Amazônia, Belém, 2010.

MIRANDA, I. P. P.; RABELO, A.; BUENO, C. R.; BARBOSA, E. M.; RIBEIRO, M. N. S. Frutos de palmeiras da Amazônia. Manaus: INPA, 2001.

MME. Ministério de Minas e Energia. Geologia, geomorfologia, pedologia, vegetação e uso potencial da terra: 1-510, Levantamento dos Recursos Naturais. Brasília: MME, 1976

NEGRELLE, R. R. B.. Attalea phalerata Mart. Ex Spreng.: Aspectos botânicos, ecológicos, etnobotânicos e agronômicos. Ciência Florestal, Santa Maria, v.25, n.4, p.1061-1066, 2015. DOI:

http://doi.org/10.5902/1980509820669

PORTELA, R. C. Q.; SANTOS, F. A. M.. Caracterização dos estádios ontogenéticos de três espécies de palmeiras: uma proposta de padronização para estudos de dinâmica populacional. Revista Brasileira de Botânica, v.34, n.4, p.523-535, 2011. DOI: http://doi.org/10.1590/S0100$\underline{84042011000400006}$

SABLAYROLLES, M. G. P.; ANDRADE, L.. Entre sabores e saberes: a importância dos quintais agroflorestais para agricultores ribeirinhos no Tapajós-PA. In: CONGRESSO BRASILEIRO DE SISTEMAS AGROFLORESTAIS. Anais. Brasília: 2009.

SALM, R.. Stem density and growth of Attalea maripa and Astrocaryum aculeatum: implication for arborescent palms distribution across Amazonian forests. Biota Neotropica, v.4, n.1, 2004. DOI: http://doi.org/10.1590/S167606032004000100003

SCARIOT, A.. Forest fragmentation effects on palm diversity in Central Amazonia. The Journal of Ecology, v.87, n.1, p.6676, 1999. DOI: http://doi.org/10.1046/j.13652745.1999.00332.x

VALADÃO, L. M.. O papel das lideranças comunitárias em projetos de saúde e ambiente: uma análise das redes sociais em comunidades do Tapajós, Pará. Dissertação (Mestrado em Desenvolvimento Sustentável) - Universidade de Brasília, Brasília, 2009.

VALENTE, R.; ALMEIDA, S.. As Palmeiras de Caxiuanã. Belém: MPEG, 2001.

WRB. World Soil Resources Reports. World reference base for soil resources. 2 ed. Rome: World Soil Resources Reports, 2006.

A CBPC - Companhia Brasileira de Produção Científica (CNPJ: 11.221.422/0001-03) detém os direitos materiais desta publicação. Os direitos referem-se à publicação do trabalho em qualquer parte do mundo, incluindo os direitos às renovações, expansões e disseminaçães da contribuição, bem como outros direitos subsidiários. Todos os trabalhos publicados eletronicamente poderão posteriormente ser publicados em coletâneas impressas sob coordenação da Sustenere Publishing, da Companhia Brasileira de Produção Científica e seus parceiros autorizados. Os (as) autores (as) preservam os direitos autorais, mas não têm permissão para a publicação da contribuição em outro meio, impresso ou digital, em português ou em tradução. 